\title{
LA ERA DE LA ECONOMIA DIGITAL -EECONOMY- Y EL REACOMODO DE LAS ESTRATEGIAS DE LOS NEGOCIOS PARA OPTIMIZAR SU COMPETITIVIDAD EN LOS MERCADOS GLOBALIZADOS
}

\author{
Econ. Nicko Alberto Gomero Gonzáles*
}

\begin{abstract}
RESUMEN
La era de la eEconomy o Economia Digital está dominando la economía mundial, las empresas están reacomodando sus estrategias, mejor dicho se están reinventando como negocio por que se han dado cuenta que si no entran al escenario digital su final como organización está muy cerca. Los mercados están más estrechados, no existe dificultad para llegar a mercado de ultramar, las carreteras reales están siendo remplazadas por carreteras virtuales que permiten el eEcommercy en tiempo virtual; los procesos de reingeniería han dejado de ser herramientas estratégicas; predominan los productos inteligentes y empresas del conocimiento. Este perfil de la eEconomy exige que los negocios se reconviertan en nuevos negocios, apuntando a la diferenciación para lograr un liderazgo sólido y sostenido. En el presente artículo, se plantean estrategias desarrolladas dentro de la economía digital, que serán de mucha utilidad para las empresas que persiguen ser mas competitivas en este tipo de economia.
\end{abstract}

\section{INTRODUCCIÓN}

Philip Kotler en su libro El Marketing se mueve afirma lo siguiente: "La Economia Digital -eEconomy- ha alcanzado un estado en el cual las compañias deben de definir con mayor firmeza su esfera de acción y la posición de sus mercados. Necesitan nuevos conceptos de márketing, nuevas capacidades $y$ conexiones que vayan mas allá de las fronteras del departamento de marketing tradicional. El marketing debe tener una mayor influencia en la estrategia empresarial de la compañia en la organización. Éste es el próximo imperativo de la transformación que determinará el estilo de la compañía en la nueva economia ${ }^{1}$.

Asimismo afirma que: "El Internet permite a la compañia expandir sus alcances geográficos de una economía exponencial. En la economia digital, las compañías no tiene que ser grandes para ser

* Docente auxiliar de la Facultad de Ciencias Contables

1 Philip Kotler-Dipac C. Jain-Suvit Maesincee. El márketing se mueve. Edit. Paidós, Buenos Aires 2002, p. 23. 
globales. Por primera vez, las compañias pequeñas pueden llegar a posibles clientes en cualquier parte del mundo... Bajo la eEconomy las empresas si quieren crecer $y$ prosperar en la economía actual, necesitarán desarrollar nuevos criterios y competencias más importantes, deben de reconsiderar y examinar sus estrategias empresariales, coordinándolas con sus estrategias de márketing, y tendrán que replantearse el papel del márketing dentro de la estrategia empresarial...»².

Bajo este contexto, sin duda, la reingenieria de procesos de negocios ya no constituye una estrategia para la nueva economia, llamada también eEconomy o economía digital. Al igual que la calidad, la reingeniería es una condición necesaria, pero no suficiente para la competitividad, puesto que el mundo, la economía y todas las reglas de negocios se encuentran en proceso de transformación. En el presente siglo las empresas deben de ir más allá de la reingeniería, su visión debe de apuntar a la transformación de la empresa, posibilitada por la tecnologia de la información (TI). La meta no sólo debe ser el control de los costos, sino la profunda transformación del servicio al cliente, la capacidad de respuesta y la innovación.

Para lograr una mayor competitividad bajo la eEconomy se debe contar con una estrategia específica basada en un rediseño del prototipo del negocio del tipo de industria a la que pertenece y la tecnología de la información permite lograr este objetivo. La economia digital exige pensar con ideas nuevas dado que las exigencias del mercado son mayores. Por ejemplo, para las industrias de manufactura la producción en masa tendrá que evolucionar hacia una producción especializada basada en bienes fisicos y en valores digitales como el Internet.

$\mathrm{El}$ crecimiento de Internet, como uno de los instrumentos más importantes de la economía digital, en ciertos sectores de la sociedad, impulsado recientemente por el desarrollo de nuevos y distintos dispositivos de enlace como teléfonos celulares, televisores convencionales, agendas electrónicas, aparatos electrodomésticos, y microcomputadoras ha contribuido, en gran medida, al aceleramiento de un proceso de transformación de los principios económicos que rigieron la actividad comercial en el siglo XX. Los cambios suscitados en materia económica que afectan ciertamente las formas de producción, mantenimiento, distribución, compra, venta, y las habilidades de la fuerza de trabajo de las organizaciones, entre otros aspectos importantes.

El siguiente trabajo apunta a explicar ¿qué es la eEconomy? ¿Cuál es su importancia en la gestión de los negocios? ¿Qué relación tiene con el eCommerce o llamado comercio virtual? El presente articulo tratará de ser claro y explícito con las ideas planteadas. Luego mostrará evidencias empíricas del avance de esta nueva economía que está revolucionando estructuralmente el mundo de los negocios.

2 Philip Kotler-Dipac C. Jain-Suvit Maesincee. El marketing se mueve, Edit. Paidós, Buenos Aires, 2002, p. 21. 


\section{FACTORES gUE VIENEN IMPULSANDO LA ECONOMÍA DIGITAL-eEconomy.}

De acuerdo al Departamento de Comercio de los Estados Unidos de Norteamérica el crecimiento que hoy observamos, de la denominada economía digital, se centra en cuatro aspectos fundamentales:

\section{a. El desarrollo constante de Internet $y$ sus tecnologias inherentes:}

En 1994, tan sólo en Estados Unidos, existian 3 millones de usuarios. Cuatro años después se superaba la cifra por 100 millones de usuarios de diversos países del mundo. Finalmente los expertos pronostican que para el año 2005, Internet alcanzará a mil millones de personas. Por supuesto que esta expansión demandará un aumento en la inversión de infraestructura tecnológica (computadoras, software, servicios y comunicaciones).

\section{b. El aumento de comercio electrónico entre empresas}

Recientemente la mayoria de las grandes empresas comenzó a utilizar a Internet para propósitos comerciales con sus respectivos socios de negocio. Pronto, algunas de ellas reportaron beneficios significantes, en materia de productividad, por el uso adecuado de las tecnologias de comunicación. Los procesos de creación, compra, distribución $y$ venta de productos $y$ servicios cambiaron positivamente $y$ de forma exponencial. Para el año 2002, se esperaban transacciones, entre negocios, por un monto mayor a los US \$300 000 millones de dólares.

\section{c. La distribución digital de bienes y servicios}

Con la llegada de la economia digital-eEconomy-algunos productos ya no tienen que ser empaquetados y distribuidos a través de una tienda física (software, noticias, música, video, etc.). Ahora una diversidad de productos y servicios son distribuidos directamente a través de Internet (boletos de avión, obras musicales, servicios personalizados de noticias, reservaciones turísticas, servicios bancarios...). Distintos sectores de diferentes industrias cambiaron la forma de operar sus negocios obteniendo beneficios significantes por el desarrollo de productos y servicios digitales.

\section{d. La proyección en la venta de bienes y servicios tangibles}

La economia digital también ha favorecido el aumento de ventas de productos convencionales o tangibles, almacenados y entregados físicamente. Aunque el porcentaje de transacciones corresponde al 1\% del total de las ventas producidas por medios convencionales (tiendas físicas), productos como computadoras, artículos electrónicos, artículos deportivos, autos, libros, y flores, entre otros, cada vez registran mayor demanda en Internet.

La intensa competencia mundial y principalmente el surgimiento de nuevas tecnologias de comunicación e información transforman cada vez más rápido a la sociedad y directa, o indirectamente, estos factores tienen un gran impacto en el actuar de las organizaciones y de los gobiernos de los distintos paises. Posiblemente somos ahora testigos del cambio más profundo desde el comienzo de la revolución industrial, y lo que contribuyó al éxito de las 
empresas en el pasado parece no tener mucho valor en el futuro. Por tal motivo resulta indispensable, para toda organización, la comprensión del "nuevo orden mundial" que deriva de una tercera revolución: "La digital". No basta con sólo mejorar las viejas formas de operación y administración de la era industrial, es importante incorporar elementos de futuro que permitan un desarrollo sustentable de acuerdo a la nueva dinámica social. "Innovar es la clave».

Internet, con su variedad de tecnologías, ha sido promotor de cambio y principal constructor de una nueva sociedad global en la era digital. Gracias a este nuevo sistema de comunicación e información cada una de las etapas comprendidas en los procesos básicos de las organizaciones, (creación, producción, comunicación, venta, servicio y control) pueden encontrar amplias posibilidades de proyección para resolver complejas operaciones de cualquier índole, simplificar o suprimir pasos innecesarios, detectar irregularidades e inventar nuevas maneras de coordinar procesos, de un modo más ágil y efectivo, en busca de la innovación.

\section{REGLAS PARA GUE LAS EMPRESAS PUEDAN ADAPTARSE Y COMPETIR BAJO LA ECONOMÍA DIGITAL}

La eEconomy - economia digitalimpulsada por la convergencia de la informática, las comunicaciones y las nuevas tecnologías, está revolucionando la forma de gestionar los negocios. El comercio electrónico - eCommerce- crece rápidamente impulsado por nuevos clientes, nuevos valores en la forma de realizar los negocios, una infraestructura tecnológica en continua expansión y un marco legal razonable. En tan sólo cinco años Internet ha pasado de ser una red de tres millones de usuarios, la mayoria residente en Estados Unidos, a ser un medio de comercio masivo, con casi 200 millones de usuarios en todo el mundo. El tráfico en Internet se duplica cada 100 días. Todo apunta a que el comercio a través de Internet sobrepasará los 700000 millones de dólares en los próximos tres años.

La convergencia tecnológica ha supuesto más de un cambio en la manera de gestionar los negocios, ha convertido en obsoletos los supuestos económicos fundamentales sobre los que se sustentaban la mayoría de empresas y transformó la economia industrial en lo que hoy denominamos economía digital.

Cinco supuestos económicos fundamentales en los que se cimentaba el éxito de compañías líder en el mercado han sucumbido: los costes de interacción $y$ transformación ya no son tan elevados, los activos fisicos no desempeñan un papel tan fundamental en la generación de la oferta, el tamaño de la empresa no condiciona los beneficios, el acceso a la información ha dejado de ser caro $y$ restringido, y ya no se necesitan varios años ni grandes capitales para establecer un negocio a escala mundial.

Estos cambios radicales en los supuestos económicos implican inmediatas y considerables amenazas para las empresas ya establecidas. Algunas de ellas comienzan a darse cuenta de que están luchando para ganar en un juego que ya no existe, y la mayoría continúa operando con estrategias 
y economías tradicionales, ajena a sus limitaciones $\mathrm{y}$ riesgos. Al mismo tiempo muchas empresas nuevas -implantadas ya en la eEconomy- están buscando la forma de mantener su éxito inicial sin seguir las viejas reglas.

Independientemente de que su empresa esté ya establecida o sea nueva, usted puede liderar la iniciativa en la emergente economía digital si comprende que las reglas y el juego de los negocios han cambiado radicalmente.

\subsection{Desintegración vertical: Los costes de interacción $y$ transformación ya no son tan elevados}

En la economía industrial los fuertes costes de interacción y transformación provocaron altos niveles de integración vertical. Para las empresas resultaba más fácil y económico poseer muchas de las piezas de la cadena de valor, desde las materias primas hasta el servicio de entrega de los productos a sus clientes. En la economia digital, las tecnologias de la información y las comunicaciones aconsejan montar una organización virtual. Mediante acuerdos con socios tecnológicamente punteros e innovadores -especializados en determinados eslabones de la cadena de valor- se pueden alcanzar nuevos niveles de calidad, flexibilidad y ahorro.

Las nuevas empresas cuyos modelos estén basados en la eEconomy deben desarrollarse continuamente, manteniendo modelos flexibles y graduales, y han de resistir la tentación de integrarse verticalmente para mejorar la calidad, la velocidad o el servicio al cliente, meditando sobre la necesidad de adquirir activos físicos o el desarrollo de cualquier actividad que no esté centrada en satisfacer las demandas principales de sus clientes.

\subsection{Los beneficios sobreactivos: Los activos físicos ya no desem- peñan el papel protagonista en la oferta de servicios de las empresas} En la economía digital la rentabilidad del mercado procedía en gran parte de los activos físicos. La propiedad, las fábricas y los equipos tenian un gran peso específico en la mayoría de los balances de las empresas. Los activos intangibles, como la marca, el $\mathrm{I}+\mathrm{D}$ y las relaciones con los clientes, aunque aportaban un valor considerable sólo existían en el contexto de los productos físicos y no solían considerarse en sí mismos una fuente de ingresos.

En la economía digital, sin embargo, la propiedad intelectual y las relaciones con los clientes han pasado a un primer plano. Al no depender ya del conjunto de activos físicos pueden ser impulsados fácilmente y a bajo coste a través de una base global de clientes. Se han convertido en una fuente independiente de ingresos y de valor.

Muchas empresas ya establecidas mantienen una importante propiedad intelectual y buenas relaciones con los clientes; pero soportan la carga de los activos físicos, que rápidamente se están convirtiendo en mercancía genérica. Las presiones en los precios están empezando a estrechar los márgenes y a disminuir las valoraciones del mercado. 
Las nuevas empresas deben impulsar continuamente el valor de la propiedad intelectual, así como el de las relaciones con los clientes -auténtico núcleo de su negociocon el fin de incrementar el volumen y los márgenes, mientras resisten la tentación de adquirir activos físicos para reducir costes a corto plazo.

\subsection{Beneficios graduales: El tamaño ya no limita los beneficios}

Tradicionalmente, la disminución gradual de los beneficios significaba que había espacio para la competencia en la mayoría de los sectores económicos. En la economía industrial, donde maximizar los beneficios significaba limitar la capacidad de producción, la única forma de satisfacer la demanda total del consumidor era a través de numerosas empresas. Por ello, tradicionalmente la disminución gradual de los beneficios significaba que había espacio para la competencia en la mayoría de los sectores económicos.

Esta regla es aplicable a la eEconomy, pero sólo para aquellos negocios basados en activos físicos. La información, la propiedad intelectual y las relaciones con los clientes no están limitadas por la capacidad de la fábrica, ya que apenas cuentan con un componente físico. De hecho, el rendimiento a escala aumenta en este nuevo entorno económico; en teoría, una empresa puede crecer sin límite, aproximando los costes unitarios a cero y aumentando exponencialmente el valor del producto para el cliente.

Como ejemplo valga una reflexión: en cualquier negocio un actor-quizá dos- pueden llegar a dominar el mercado. Una vez que una empresa consigue un sólido liderazgo seguirá creciendo hasta situarse en una posición de insuperable dominio. En este contexto económico, las empresas ya establecidas pueden rápidamente quedarse detrás y no recuperarse jamás. Entre tanto, las nuevas empresas deben redefinir constantemente las ofertas que realizan a sus clientes con el fin de reconducir el volumen y rebajar los costes resistiendo la tentación de competir exclusivamente en el precio.

\subsection{Acceso a la información: El acceso a la información ha dejado de ser caro y restringido}

En la economía industrial, la información para los compradores y vendedores era con frecuencia cara y difícil de obtener. Los consumidores tenían pocas fuentes de información sobre productos, aparte de las que le proporcionaba la publicidad o la recomendación de un amigo. Los fabricantes y minoristas llevaban a cabo investigaciones de mercado para conocer las necesidades y comportamientos de los compradores, sin posibilidad de segmentación.

Bajo la eEconomy la información es más barata y fácil de obtener. Las empresas están comprendiendo que los clientes son cada vez más exigentes debido a la gran cantidad de ofertas y al fácil acceso a una información más completa. En este nuevo entorno están bajo la presión que supone crear continuamente ofertas innovadoras y valiosas para contrarrestar la competencia en precio, que está al alcance de un solo click del ratón de sus clientes. 
¿Cuál es entonces la buena nueva? La respuesta está en que en la economía digital existen enormes oportunidades para todos. Los fabricantes y proveedores de servicios pueden recopilar información sobre los clientes y utilizarla para proporcionar a cada uno de ellos exactamente lo que desea; para facilitarles de manera personalizada unos productos y servicios que nunca antes habian imaginado.

\subsection{El tiempo para llegar al mercado: Ya no se necesitan varios años ni grandes capitales para establecer un negocio con presencia a escala mundial}

En la economía industrial abrir nuevos mercados representaba un gran esfuerzo. Llevaba años realizar estudios de mercado, construir y desplegar activos físicos, identificar y elaborar inventarios específicos, $y$ generar la capacidad necesaria de producción y venta. En la actualidad se pueden abrir nuevos mercados de un día para otro. Los canales virtuales pueden utilizarse para buscar fuentes de productos y servicios y para promocionar, vender y dar soporte a los mismos... y todo ello de forma más rápida y barata que investigar si la expansiôn es una buena idea.

En consecuencia, las empresas ya establecidas están viendo cómo los nuevos competidores se introducen rápidamente en los mercados logrando así una apreciable cuota de mercado. Mientras tanto, las nuevas empresas deben continuar innovando sus ofertas para alcanzar diferentes mercados, reforzar sus marcas comerciales y ampliar su alcance mundial.
El éxito en la eEconomy requiere más que la creación de una página web, el lanzamiento de canales virtuales, la adecuación de los procesos comerciales de forma electrónica o el establecimiento de negocios independientes por Internet. Lo que realmente exige es un examen constante de todo su modelo de negocio. Por ejemplo, en la economia industrial las empresas de mayor éxito identificaron primero sus negocios básicos y después buscaron los clientes adecuados. En la economía digital la fórmula es a la inversa: primero se ha de fijar la atención en los deseos de los clientes y después configurar el negocio para satisfacer sus demandas. Este cambio sustenta la primera de las preguntas básicas que usted debe hacer para revalorizar su modelo de negocio:

- ¿Qué es lo que los consumidores desean comprar?

- ¿En qué negocios debemos estar presentes?

- ¿Cuál es la propuesta más efectiva a corto, medio y largo plazo?

- ¿Qué papeles debemos desempeñar -fabricar, vender o dar servicio- y quiénes son nuestros clientes?

- ¿Cuál es el mejor modelo de funcionamiento?

- ¿Con quién debemos asociarnos?

Las respuestas a estas preguntas, sin duda, le ayudarán a alcanzar el éxito empresarial en la eEconomy.

\section{DOCE TEMAS DE LA NUEVA ECONOMÍA DIGITAL}

Virtualidad, economía y conocimiento son los vértices del 
cambio al nuevo siglo. En los próximos años veremos consumarse la transición de una economía de capital a una economia basada en el conocimiento.

La sintesis de los 12 temas de una economía digital plantea un punto de partida obligado en cualquier perspectiva de análisis que centremos alrededor de la economía en general $y$ del desarrollo empresarial en particular a la luz de los cambios tecnológicos actuales.

Doce son los temas de la economía digital.
i. Globalización
ii. Conocimiento
iii. Innovación
iv. Digitalización
v. Virtualización
vi. Convergencia
vii. Interconexión en red
viii. Molecularización
ix. Orientación a clientes
x. Inmediatez
xi. Desintermediación
xii. Discordancia

\section{La nueva economía es una economia global}

- Una economía basada en conocimiento volatiliza las fronteras.

- Los productos y servicios adquieren gran movilidad buscando explotar las ventajas comparativas de bloques y regiones.

- La globalización y la tecnología actúan como causa y efecto de un mismo proceso de desarrollo mundial.
- El mundo académico y empresarial trabajan en la conformación de empresas virtuales, redes de respuesta comercial, firmas sin fronteras $y$ organizaciones globales.

II. La nueva economía es una economia de conocimiento

- Las ideas de los individuos, la información y la tecnología se convierten en parte de productos (confecciones, tarjetas, casas, autos, televisores, teléfonos $y$ otros productos inteligentes).

- Los activos claves de la organización radican en los trabajadores del conocimiento (capital intelectual)

- La competitividad de una organización está en la capacidad de aprender más rápido que los competidores o lo que es lo mismo, el aprendizaje organizacional permanente se convierte en la única ventaja competitiva sostenible.

\section{La nueva economía estará} basada en la innovación

- La actividad de innovación se compromete con la renovación continua de productos, sistemas, procesos, mercadotecnia y manejo del personal.

- Ciclos de vidas de los productos y servicios muy cortos.

- En una economía de innovación la imaginación y creatividad humana es la principal fuente de valor.

- Aprender a comprender las necesidades de los clientes, de sus clientes, es innovar y entrar en la cadena de valor sostenible. 
IV. La nueva economia es digital

- Los nuevos medios, la autopista de la información y la nueva economía se construyen sobre la base del bit.

- Los medios de transmisión, reproducción, visualización y presentación de la información convergen hacia un mundo digital.

- Nuevos conceptos de trabajo y capacidades creativas se desarrollan a partir de la digitalización de servicios profesionales (diseño asistido por computador, trabajo en grupo medido por computador, laboratorios virtuales, etc.)

v. Al cambiar la información de análogo a digital los elementos físicos se tornarán virtuales, cambiando así el metabolismo de la sociedad

- Nuevos y vastos campos de la actividad socioeconómica, donde la información es un factor crítico, pasan a la virtualidad como plataforma de desempeño:

- Trabajo virtual.

- Educación virtual.

- Congreso virtual.

- Centro Comercial Virtual, etc.

VI. El sector económico más importante se sustenta en la convergencia de las industrias de la informática, las comunicaciones y la información

- El desarrollo del hardware y del ancho de banda de las comunicaciones abre el camino para que las nuevas ganancias estén en el sector de los servicios de información donde se crea valor para los consumidores.

- La visión hacia la convergencia ofrece nuevas ventajas e impactará en la aparición de nuevas empresas y alianzas estratégicas para la nueva economía.

\section{La nueva economía es una economía en red}

- Se crea un nuevo modelo operativo de los negocios a través de la capacidad de segmentación exclusiva para un mercado de un solo cliente.

- La nueva empresa interconectada permite a las PyMES superar las ventajas (economía de escala y acceso a los recursos) de las grandes compañias y elimina la burocracia, la jerarquía innecesaria y la incapacidad de cambiar.

- Se reconfiguran las relaciones de las empresas y se produce un incremento dramático del outsourcing.

VIII. Se desagrega la economía hacia formas moleculares más dinámicas y flexibles. Lo masivo se convierte en molecular

- La reingeniería de procesos: inicio de una transformación hacia formas más productivas de orientación al cliente.

- El trabajador del conocimiento (molécula de la organización) funciona como una unidad de negocios, creando e incorporando valor a la producción. 
- Los mercados en masa son sustituidos por un mercado activo con nichos importantes para la pequeña y mediana empresa con alta funcionalidad y orientación rápida a los cambios de los clientes.

IX. Las empresas, funciones y personas necesitarán subir a la cadena de crear valor o desaparecerán

- Las actividades cuya esencia está en la repetición de señales de información tienden a desaparecer presionadas por la interconexión en red entre consumidores y productores de bienes y servicios.

La intermediación de información se reconfigura hacia la agregación de valor en la transacción a través de un profundo análisis de productores y consumidores.

$X$. Se reduce la brecha entre consumidores y productores

- Se particulariza el mercado y la producción masiva se sustituye por la producción orientada a clientes.

- La participación activa de los consumidores en ambientes de comunicación electrónica, los hace partícipes de procesos de diseño y producción.

- Al aumentar el contenido de información y conocimiento de los productos y servicios, las empresas tienden a convertirse en productores de infotecnología.
XI. En una economía basada en bits, la inmediatez se convierte en impulsor y variable clave en la actividad económica y de éxito de las empresas

- La empresa en tiempo real.

- Los sistemas just in time cambian la naturaleza de muchos negocios.

- El tiempo de respuesta es una variable crítica de supervivencia en el negocio.

- El intercambio electrónico de documentos plantea la señal de una oleada de nuevas formas de hacer comercio y un metabolismo de los negocios hacia un tiempo real.

\section{Problemas sociales sin precedentes causan traumas y conflictos masivos.}

- Fuertes presiones para la descentralización del poder económico y político.

- Poca movilidad laboral entre una economía industrial y una basada en el conocimiento.

- Los trabajadores del conocimiento requieren de la motivación y una relaciones de equipo confiables para ser efectivos.

- La tendencia de los que no tengan acceso a la nueva infraestructura del conocimiento es quedarse cada vez más a la saga.

- Se reconfiguran los sistemas educativos.

\section{INTERNET EN CIFRAS}

El desarrollo que ha registrado Internet ha sido fenomenal, dejó de 
ser la red particular de instituciones cientificas, de educación y de algunas dependencias de gobierno de diversos países para convertirse en la red pública más grande del mundo, con un alcance aproximado de 300 millones de usuarios en más de 170 países.

Según las últimas cifras publicadas por la Internet Society (ISOC), organismo internacional promotor de Internet, existen aproximadamente $9,560,866$ sitios web, con información diversa de cualquier parte del mundo. Y de acuerdo a un estudio presentado por Euro-Marketing, los 5 idiomas predominantes en Internet, además del inglés son: Japonés 7,1\%, Español 6,2\%, Chino 5,4\%, Alemán $5,0 \%$, Francés 3,9\%.

Durante 1998 se registró una de las últimas propuestas masivas de conexión de diversos países denominados en desarrollo. Sin embargo, bajo la óptica de algunos estudiosos del fenómeno, este esfuerzo no ha sido suficiente para
Figura 1. Número de máquinas conectadas a Internet

Millones de máquinas conectadas a internet

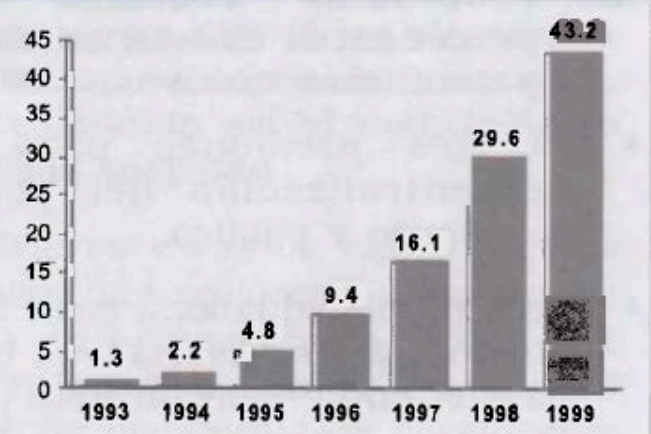

Figura 2. Distribución de máquinas conectadas a Internet

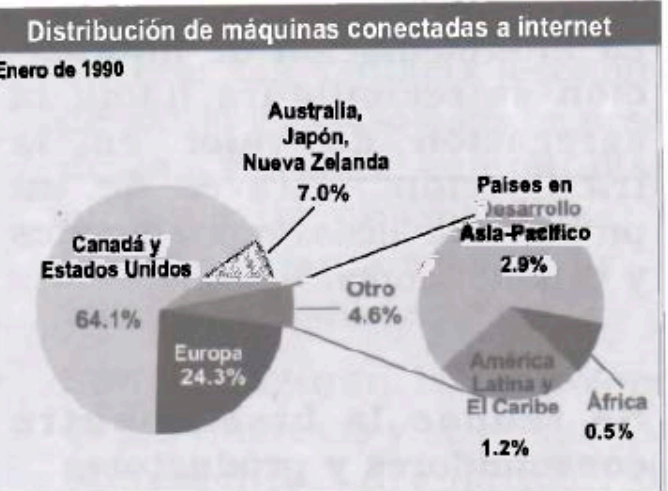

Fuente: Network Wizards dsiponible en línea: http://www.nw.com (1999)

democratizar el medio. La participación de los países en desarrollo es casi nula en comparación con algunos países desarrollados. Según Network Wizards, el $97 \%$ de los hosts, o computadoras conectadas a Internet, reside en los países desarrollados.

Como se muestra en las gráficas anteriores los países en desarrollo mantienen una participación limitada en Internet, principalmente como consecuencia de la baja oferta en sus respectivos mercados. No obstante este problema, con motivo de algunas iniciativas de diversos sectores de la sociedad, destacan ahora, de forma sorprendente, algunos países en desarrollo como Argentina, Brasil, Chile, Colombia, México y Venezuela, en el caso particular de Latinoamérica. Algo similar sucede con la India y otros países de la región Asia-Pacífico. El caso específico de Brasil y México es importante de señalar. Brasil ocupa el lugar 13 en número de hosts, mientras que México registra 
el lugar 15 a nivel mundial, según un estudio de productos convencionales o tangibles, almacenados y entregados fisicamente. Aunque el porcentaje de transacciones corresponde al $1 \%$ del total de las ventas producidas por medios convencionales (tiendas fisicas), productos como computadoras, artículos electrónicos, artículos deportivos, autos, libros, y flores, entre otros, cada vez registran mayor demanda en Internet.

La intensa competencia mundial y principalmente el surgimiento de nuevas tecnologias de comunicación e información transforman cada vez más rápido a la sociedad, y directa o indirectamente, estos factores tienen un gran impacto en el actuar de las organizaciones y de los gobiernos de los distintos paises. Posiblemente somos ahora, testigos del cambio más profundo desde el comienzo de la revolución industrial, y lo que contribuyó al éxito de las empresas en el pasado parece no tener mucho valor en el futuro. Por tal motivo resulta indispensable, para toda organización, la comprensión del "nuevo orden mundial" que deriva de una tercera revolución: "La digital». No basta con sólo mejorar las viejas formas de operación y administración de la era industrial, es importante incorporar elementos de futuro que permitan un desarrollo sustentable de acuerdo a la nueva dinámica social. "Innovar es la clave».

Internet, con su variedad de tecnologías, ha sido promotor de cambio y principal constructor de una nueva sociedad global en la era digital. Gracias a este nuevo sistema de comunicación e información, cada una de las etapas comprendidas en los procesos básicos de las organizaciones, (creación, producción, comunicación, venta, servicio y control), pueden encontrar amplias posibilidades de proyección para resolver complejas operaciones de cualquier indole, simplificar o suprimir pasos innecesarios, detectar irregularidades, e inventar nuevas maneras de coordinar procesos, de un modo más ágil y efectivo, en busca de la innovación.

\section{ECONOMÍA DIGITAL. EXPE- RIENCIA CHILENA}

La Economía Digital en Chile habria alcanzado una importancia económica equivalente a US\$ 5.532 millones en el año 2000, de acuerdo a un estudio de la Cámara de Comercio de Santiago (CSS) dado a conocer durante el seminario "La Economia Digital 2001". Dicha cifra comprende las ventas relacionadas a la industria de las Tecnologías de la Información (TI) y a su reciente convergencia con las comunicaciones.

Para establecer la medición, el Departamento de Estudios de la CCS construyó indicadores de ventas en cinco grandes subsectores: infraestructura TI y de comunicaciones, telecomunicaciones, aplicaciones de negocios, servicios (como medios de pago, consultoria, research y contenidos, entre otros), y transacciones de comercio electrónico.

De acuerdo al estudio, que forma parte de La Economia Digital en Chile 2001 , dado a conocer ante más de 400 empresarios, el sector crecerá a tasas promedio del $30 \%$ en los próximos años, llegando a una significancia superior a los US\$ 17000 millones el año 2004.

E1 componente de mayor crecimiento será el comercio electrónico, que en el año 2000 generó ventas por US\$ 465 millones, 5 veces superiores a las de 1999, explicado mayoritariamente por los negocios business to business (B2B) Asimismo, se proyecta un acelerado crecimiento en los próximos cinco años, hasta superar los US\$ $10 \mathrm{mil}$ 
millones el 2004. Pese a este acelerado crecimiento, se estima que el comercio electrónico explicará un $2 \%$ de las ventas minoristas en dicho periodo, mientras que el B2B canalizará el $6 \%$ de las transacciones totales entre empresas.

En otro ámbito, la CCS desarrolló un modelo para proyectar el impacto que tendrá la Economía Digital sobre el crecimiento potencial del país, concluyendo que éste permitirá acelerar en un $0,6 \%$ anual promedio la expansión del PIB nacional en los próximos 5 años.

Finalmente, se estimaron los posibles efectos de la revolución tecnológica sobre la generación de empleo, proyectándose una creación neta de 130 mil nuevos puestos de trabajo para los próximos cinco años, lo que equivale a poco más de un $2 \%$ de la fuerza de trabajo en Chile.

\section{CONCLUSIONES}

1. Los instrumentos tradicionales de gestión empresarial, como son la calidad, la reingeniería, justo a tiempo, ya no son suficientes para que un negocio mantenga el liderazgo en los mercados globalizados. La eficacia del ajuste de los costos, para mantener los márgenes, en donde cada consumidor se ha convertido en un mercado objetivo, ha dejado de ser un instrumento de alta eficacia para que las empresas apunten a lograr utilidades sostenidas.

2. En la era de la Economía Digital -eEconomy- las empresas para ganar liderazgo tendrán que reacomodar sus estrategias empresariales. Las nuevas estrategias necesariamente deben de estructurase en función de la convergencia tecnológica que todo negocio debería de desarrollar en este nuevo escenario económico: la informática, las comunicaciones $y$ la información.

3. Bajo la economia digital, el tamaño de los negocios no garantiza su supremacia en el mercado; empresas pequeñas, inteligentes y con elevado manejo de la informática, las comunicaciones y la información se pueden convertir en una organización de mayor valor que una empresa de gran envergadura, que se encuentra enfrascada en los paradigmas tradicionales de gestión de negocios.

4. El Internet, más que un instrumento de comunicación, se ha convertido en un medio para llegar a mercados con fines de hacer negocios. Las transacciones económicas por este medio, llamada eCommerce, han originado además el quiebre de ciertos paradigmas de gestión de negocios, como el de pensar que llegar a mercados externos es costoso; con este medio no sólo se puede lograr vender en mercados europeos o africanos, sino contratar especialistas altamente tecnificados en estos países, por cierto sin que éstos se muevan de su país de origen, que, como es obvio, abarata los costos de gestión.

5. La eEconomy ha roto el paradigma de que producir en escala implica abaratar los costos y maximizar beneficios, bajo este nuevo escenario económico, cada cliente es un mercado exclusivo, cada consumidor es un segmento especial, que merece atención especial y preferencial, es decir, que los negocios deben estar preparados para atender las necesidades y cubrir en exceso las expectativas de cada consumidor. Los clientes se han 
convertido en los elementos estratégicos de los negocios, cuyas opiniones "Vuelan" por la supercarretera de la información.

6. Los negocios deben estar preparados para responder en tiempo virtual las exigencias del mercado. Los niveles administrativos y operativos, deben de introducirse a un tipo de cultura empresarial, en donde prime la eficacia como producto del trabajo horizontal.

7. Los negocios basados en el conocimiento, especializados en la producción de productos inteligentes, especializados y a veces únicos, utilizan la eEconomy para lograr la diferenciación de sus productos, medio que le permite obtener posicionamientos sólidos y sostenidos en los mercados globalizados, siendo uno de los recursos para tal fin la inteligencia cuya aplicación, acompasada con los demás recursos de la eEconomy, origina que el negocio maximice su valor como organización. Lo cual ha originado además que los recursos reales ( activos fijos reales) tengan una depreciación acelerada en el campo tecnológico, económico y financiero.

\section{ANEXO}

Diferencia entre empresas convencionales y las basadas en el conocimiento

\begin{tabular}{|l|l|}
\hline \multicolumn{1}{|c|}{ CONVENCIONALES } & \multicolumn{1}{|c|}{ CONOCIMIENTO } \\
\hline Buscar producir riquezas & Buscan producir riquezas \\
\hline Ofertan productos y servicio comunes & $\begin{array}{l}\text { Ofertan productos y servicio } \\
\text { especializados (a veces unnicos) }\end{array}$ \\
\hline Se compite por escalas y costos & $\begin{array}{l}\text { Se compite por diferenciación en } \\
\text { productos v servicios }\end{array}$ \\
\hline $\begin{array}{l}\text { La creación de conocimientos no se } \\
\text { incorpora al costo }\end{array}$ & $\begin{array}{l}\text { Mucho conocimiento incorporado en el } \\
\text { costo v el precio }\end{array}$ \\
\hline Poco gasto en I+D & Alto gasto en I+D \\
\hline $\begin{array}{l}\text { Comercia esencialmente bienes tangibles } \\
\text { (productos) }\end{array}$ & $\begin{array}{l}\text { Alta componente de negociación sobre } \\
\text { intangibles }\end{array}$ \\
\hline $\begin{array}{l}\text { Productos y procesos menos protegidos } \\
\text { por patentes (se protege por know how } \\
\text { confidencial) }\end{array}$ & Amplio uso de la protección por patentes \\
\hline $\begin{array}{l}\text { Orientada a utilidades inmediatas } \\
\text { Generalmente evitan el riesgo }\end{array}$ & $\begin{array}{l}\text { Orientada a utilidades inmediatas y } \\
\text { futuras }\end{array}$ \\
\hline $\begin{array}{l}\text { Recursos humanos de calificación media } \\
\text { Recursos humanos reemplazables con la } \\
\text { fuerza laboral disponible }\end{array}$ & Recursos humanos de alta calificación \\
\hline $\begin{array}{l}\text { Tratamiento de los recursos humanos humanos irremplazables } \\
\text { como costo variable }\end{array}$ & $\begin{array}{l}\text { Tratamiento de los recursos humanos } \\
\text { como costo fijo }\end{array}$ \\
\hline
\end{tabular}

El desplazamiento de las empresas es continuo de izquierda a derecha de la tabla en la medida que las empresas convencionales incorporan innovaciones que se vuelven decisivas y comienzan a operar como empresa basadas en conocimiento. 
Figura 3. Alcance del Internet por región: Año 2000

\begin{tabular}{|c|c|}
\hline Total usuarios & 275.54 Millones \\
\hline África & 2.46 Millones \\
\hline Asia/Pacifico & 54.90 Millones \\
\hline Europa & 71.99 Millones \\
\hline Medio Oriente & 1.29 Millones \\
\hline Canadá y Estados Unidos & 136.06 Millones \\
\hline América del Sur & 8.79 Millones \\
\hline
\end{tabular}

Fuente: NUA Internet Surveys, (2000)

Figura 4. Crecimiento de los paises con mayor número de hosts en Internet

\begin{tabular}{|l|l|l|l|l|l|l|l|l|l|l|}
\hline & & Ene-96 & Jul-96 & Ene-97 & Jul-97 & Ene-98 & Jul-98 & Ene-99 & Jul-99 & Ene-00 \\
\hline 1 & Japón & 269327 & 496427 & 734406 & 955688 & 1168956 & 1352200 & 1687534 & 2072529 & 2636541 \\
\hline 2 & $\begin{array}{l}\text { Reino } \\
\text { Unido }\end{array}$ & 451750 & 579492 & 591624 & 878215 & 987733 & 1190663 & 1423804 & 1599497 & 1901812 \\
\hline 3 & $\begin{array}{l}\text { Estados } \\
\text { Unidos }\end{array}$ & 233912 & 432727 & 587175 & 825048 & 1076583 & 1302204 & 1562391 & 1555882 & 1875663 \\
\hline 4 & Alemania & 452997 & 548168 & 721847 & 875631 & 994926 & 1154340 & 1316893 & 1426928 & 1702486 \\
\hline 5 & Canadá & 372891 & 424356 & 603325 & 690316 & 839141 & 1027571 & 1119172 & 1294447 & 1669664 \\
\hline 6 & Australia & 309562 & 397460 & 514760 & 707611 & 665403 & 750327 & 792351 & 907637 & 1090468 \\
\hline 7 & Molanda & 174888 & 214704 & 270521 & 341560 & 381172 & 514660 & 564129 & 637591 & 820944 \\
\hline 8 & Francia & 137217 & 189786 & 245501 & 292096 & 333306 & 431045 & 488043 & 653686 & 779879 \\
\hline 9 & Italia & 73364 & 113776 & 149595 & 211966 & 243250 & 320725 & 338822 & 393627 & 658307 \\
\hline 10 & Finlandia & 208502 & 277207 & 283526 & 335956 & 450044 & 513527 & 546244 & 577029 & 631248 \\
\hline 11 & Taiwan & 25273 & 30645 & 34650 & 40706 & 176836 & 103661 & 308676 & 424209 & 597036 \\
\hline 12 & Suecia & 149877 & 186312 & 232955 & 284478 & 319065 & 380634 & 431809 & 515031 & 594627 \\
\hline 13 & Brasil & 20113 & 46854 & 77148 & 68685 & 117200 & 163890 & 215086 & 310138 & 446444 \\
\hline 14 & España & 53707 & 62447 & 110041 & 121823 & 168913 & 243436 & 264245 & 302457 & 415641 \\
\hline 15 & México & 13787 & 20253 & 29840 & 35238 & 41659 & 83949 & 112620 & 224239 & 404873 \\
\hline
\end{tabular}

Nota: La siguiente abstracción de un estudio general sobre el número de usuarios de Internet demuestra la importancia que registra actualmente este nuevo medio de comunicación en el ámbito global. 


\section{BIBLIOGRAFÍA}

1. DON TPASCOTT. La economía Digital Edit. Mc GrawHill. Colombia, 1999.

2. PHILP KOTLER-DIPAK JAIN. SUVIT MAESINCEE. El marketing se mueve, Edit Paidós, España, 2002.

3. GARCÍA GONZALES FIDEL Gestión del Conocimiento en Tiempos de Economía Digital. Universidad de Camaguey-Cuba.

4. Cámara de Comercio de Santiago de Chile. Economía Digital en Chile, 2000.

5. TOSCANO César. Uso estratégico de la tecnología de la Información. Toscano Management Consulting 2000

6. VILASECA-REQUENA KORDI. Mi-diendo la economía Digital: una aproximación $a$ un indicador de demanda de las TIC para los EE. UU. Universidad Oberta de Catalunya, 2000.
7. CORZO CARLOZ. Planeamiento Estratégico del Comercio electrónico, Symetry Sistems-Comon Com, 2000, Lima

8. La Economia Digital en Chile. www.ccs.cl/html

9. Derecho y Economía digital-2000. www.it-.cenit.org.ar/seminaries

10 Los dividendos de la economía digital -2001. Revista del Centro de Comercio Internacional. ww. Forumcomercio.or.

11. Comercio Electrónico Digital. www.e global.es/acded.htm

12. Gestión del conocimiento en tiempos de Economía Digital. Un modelo de Competitividad académico aplicado a los entornos empresariales. www.Gestióndel conocimiento.com (Footnotes) 